\title{
Analysis of a Chaotic Memristor Based Oscillator
}

\author{
F. Setoudeh, ${ }^{1}$ A. Khaki Sedigh, ${ }^{2}$ and M. Dousti ${ }^{1}$ \\ ${ }^{1}$ Department of Electrical and Computer Engineering, Science and Research Branch, Islamic Azad University, \\ Tehran 14515-775, Iran \\ ${ }^{2}$ Department of Electrical Engineering, Industrial Control Center of Excellence, K. N. Toosi University of Technology, \\ Tehran 14317-14191, Iran \\ Correspondence should be addressed to F. Setoudeh; f_setoudeh@srbiau.ac.ir
}

Received 10 August 2013; Revised 2 October 2013; Accepted 20 October 2013; Published 22 May 2014

Academic Editor: Carlo Bianca

Copyright (C) 2014 F. Setoudeh et al. This is an open access article distributed under the Creative Commons Attribution License, which permits unrestricted use, distribution, and reproduction in any medium, provided the original work is properly cited.

A chaotic oscillator based on the memristor is analyzed from a chaos theory viewpoint. Sensitivity to initial conditions is studied by considering a nonlinear model of the system, and also a new chaos analysis methodology based on the energy distribution is presented using the Discrete Wavelet Transform (DWT). Then, using Advance Design System (ADS) software, implementation of chaotic oscillator based on the memristor is considered. Simulation results are provided to show the main points of the paper.

\section{Introduction}

The study of nonlinear systems is an important research topic for scientists and researchers. One of the complex types of behavior that can be observed in these systems is the chaotic behavior. Chaos is widely available in engineering and the natural systems. Chaos phenomenon is completely deterministic and specific to nonlinear systems. In fact, chaos theory is a branch of mathematics and physics related to systems that their dynamic represents a very sensitive behavior to changes in the initial values so that their future behavior is not predictable anymore; these systems are called chaotic systems which are of nonlinear ones. Nowadays, there are examples of potential benefits of the chaotic behavior which make a lot of engineers and researchers attend it. Bianca and Rondoni proposed the analytical and numerical investigations of Ehrenfest gas, which is a billiard model with an electric field and a Gaussian thermostat [1]. Bianca considers chaotic behavior of two thermostat billiards: the nonequilibrium Ehrenfest gas (NEEG) and the pump model (PM) [2].

One of these nonlinear systems occurs in that chaos is sinusoidal oscillator circuits [3].

In the theoretical circuit studies, there are certain fundamental elements in the circuit, including resistance, capacitance, and inductance. Resistance is the relationship between the current and the voltage, capacitance is the relationship between the charge and the voltage, and also inductance is the relationship between the magnetic charge and the current. These elements have two inactive terminals used in different circuits and it is known that no information is saved in them. This point may seem unimportant, but in fact it is considered as a fundamental principle in circuits which is indicative of a charge pattern in electronics [4]. For example, it is sometimes needed to have elements like resistance for a kind of calculation or process in a circuit. These four elements constitute four fundamental elements of a circuit. Memristor includes two-terminal circuit elements and is an abbreviation of the memory resistor [5]. In terms of hardware, a microscopic tool is available that can keep its previous electrical conditions. Hence, the temporary memory can be preserved even after power cut and it consumes energy only when we intend to read its information. This element can replace transistors in some circuits and occupy less space [6] and is made in a nanoscale, while its resistance is dependent on the domain, polarity, and voltage time used in it [7]. When voltage is cut, memristor can remember the last resistance produced until a voltage with different amounts of polarity is applied to it [6]. In recent years, researchers have done a lot of work on designing an oscillator using memristor [811]. For instance, memristor has been applied in designing Chua's oscillator [10]. Memristor depends on a group of 


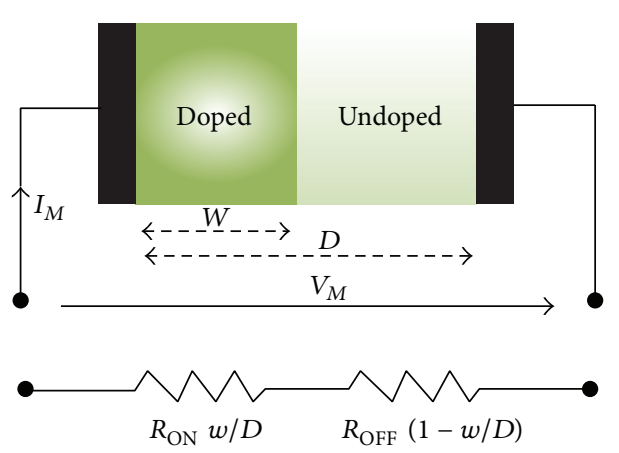

Figure 1: Physical model of memristor.

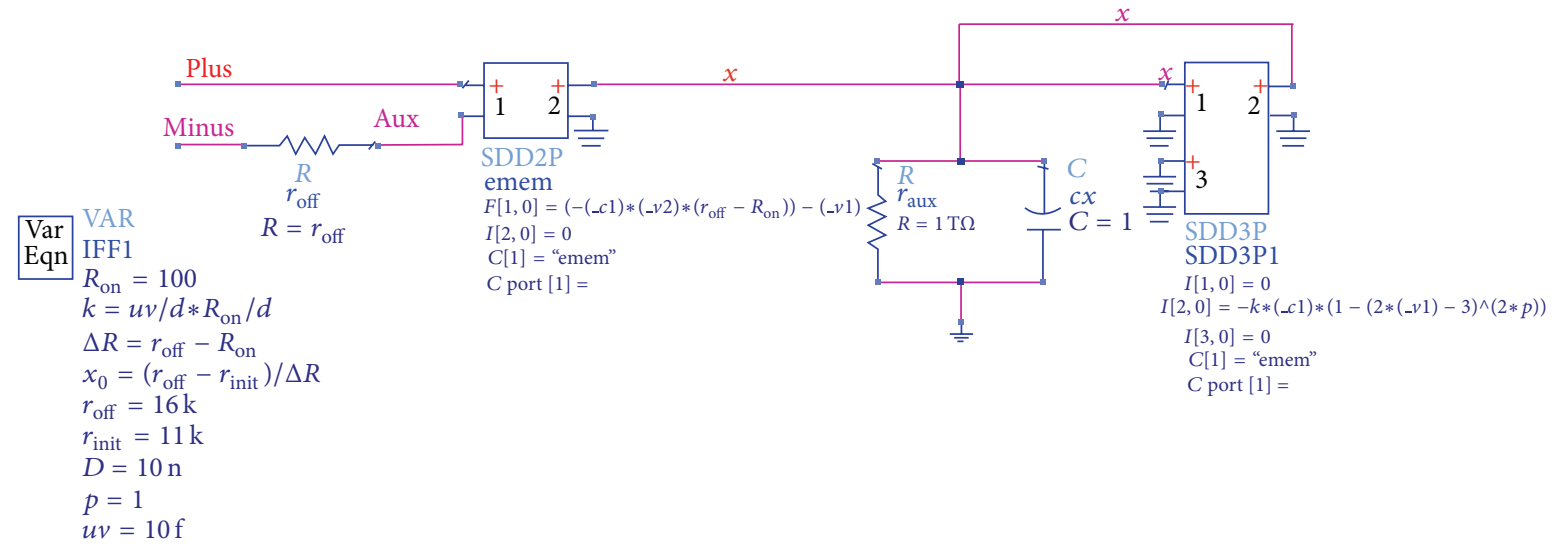

FIgURE 2: Schematic of the memristor ADS implementation.

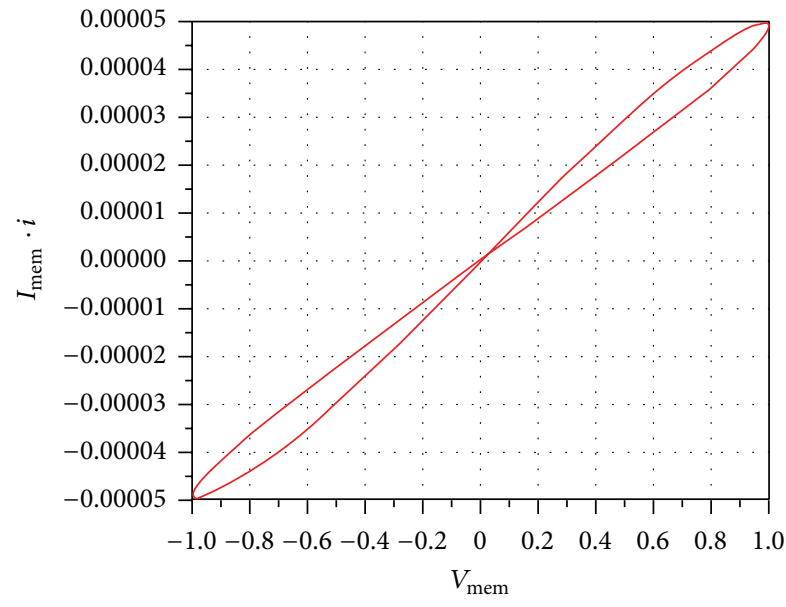

FiguRE 3: Memristor current versus voltage (hysteresis loop).

memristive systems that can display a chaotic behavior in a circuit due to a series of nonlinear properties [12]. In recent years, many researchers have attempted analyzing the chaotic behavior of circuits, including memristors. For example, the chaotic behavior of a memristor in a Chua's oscillator circuit is studied in [11]. Chua's oscillator circuit is the simplest chaotic circuit that in its structure, a nonlinear

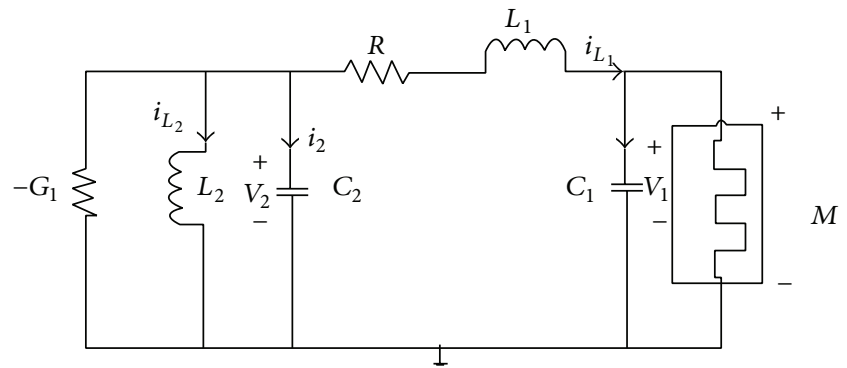

FIgURE 4: The schematic of Chua's circuit.

element called Chua's diode was replaced with a memristor [13], where a segmented linear model of a memristor is used and also Lyapunov's exponent tool is employed to identify chaos [11]. The chaotic behavior of a memristor has also been studied in other circuits of orders 3 and $4[9,14]$. However, no mathematically oriented chaos analysis is presented. In this paper, a hyperchaotic oscillator based on a memristor is considered and the chaos phenomenon is studied and analyzed accurately in this circuit considering a nonlinear model of the memristor. Then a methodology based on the energy distribution is presented using the Discrete Wavelet Transform (DWT) for chaos analysis. 

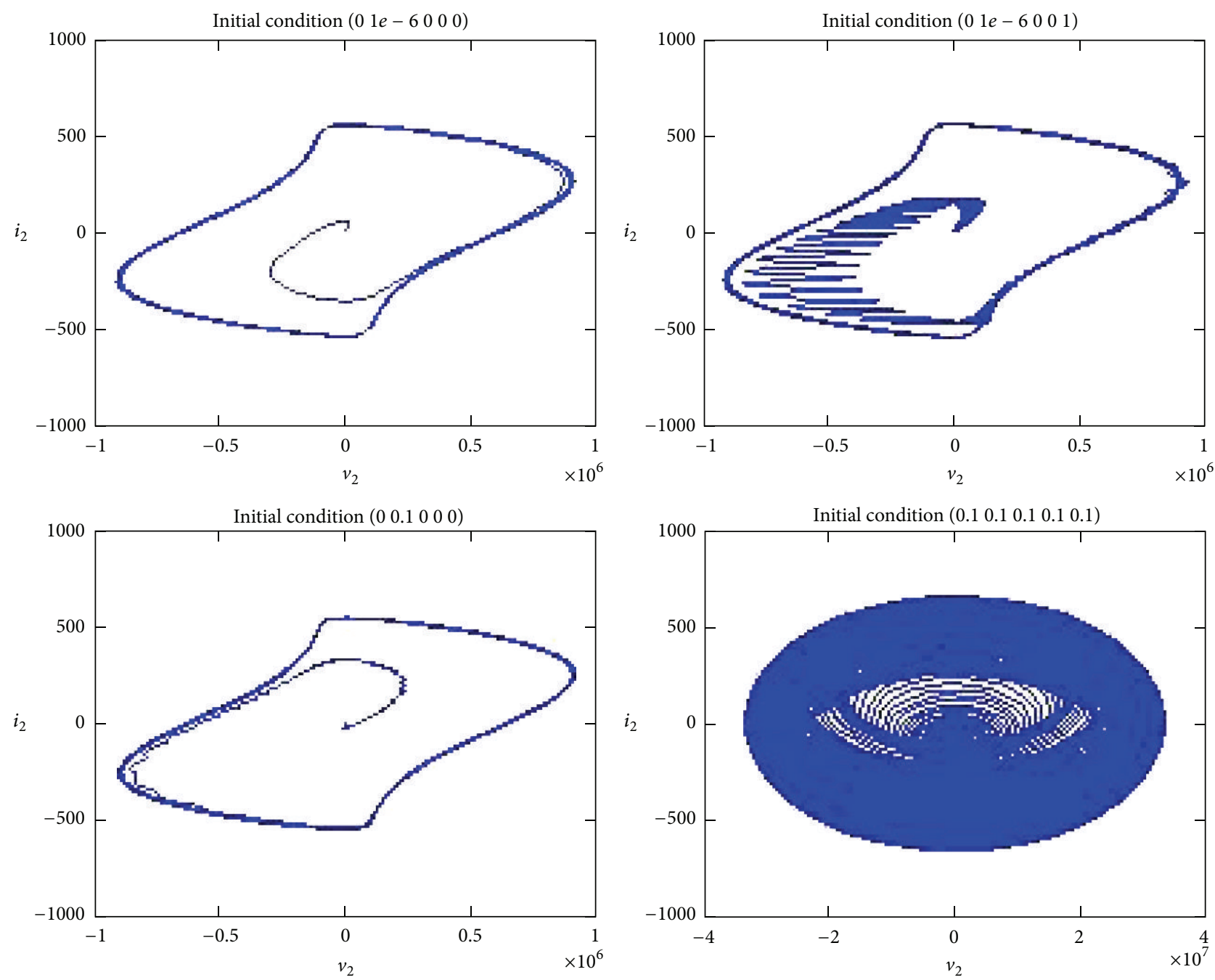

FIGURE 5: Transient chaotic attractor in different initial conditions.

\section{Memristor Preliminaries}

2.1. Memristor Principles. Memristor (memory resistor) is a microscopic tool that can keep its previous electrical conditions and can therefore preserve the temporary memory even after a power cut. According to the definition, memristor is determined by $g(q, \varphi)=0$ relation [4]. If this relation is expressed only as a one-variable function from charge (flux linkage), memristor will be called charge-controlled. Two-terminal voltage of the charge-controlled memristor is acquired from the following relation [5]:

$$
d \varphi(q)=M(q) d q
$$

where $q$ is the electric charge, $\varphi$ is the magnetic flux, and $M(q)$ is a part of the resistance and is called the memristance. It is known that magnetic flux is the integration of voltage and charge is the integration of current; then memristance can be defined as [5]

$$
M(q(t))=\frac{d \varphi / d t}{d q / d t}=\frac{v(t)}{i(t)} .
$$

Similarly, the current of the controlled memristor with flux is acquired from the following relation [9]:

$$
i(t)=\frac{d q}{d t}=\frac{d q}{d \varphi} \frac{d \varphi}{d t}=W(\varphi(t)) v(t) .
$$

So that

$$
W(\varphi(t))=\frac{d q(\varphi)}{d \varphi},
$$

where $W(q)$ is called the memductance since it includes some conductivity.

It is readily observed that the value of the memductance at $t=t_{0}$ depends on the memristor (voltage) current time integral from $t=-\infty$ to $t=t_{0}$. Hence, while the memristor at a specific time $\left(t=t_{0}\right)$ behaves as a normal resistance, its resistance (conductivity) is dependent on the past current (voltage) of the memristor.

2.2. Memristor Implementation. The physical structure of the memristor consists of a thin-layer film of two-layer titanium 
Initial condition $(01 e-5000)$

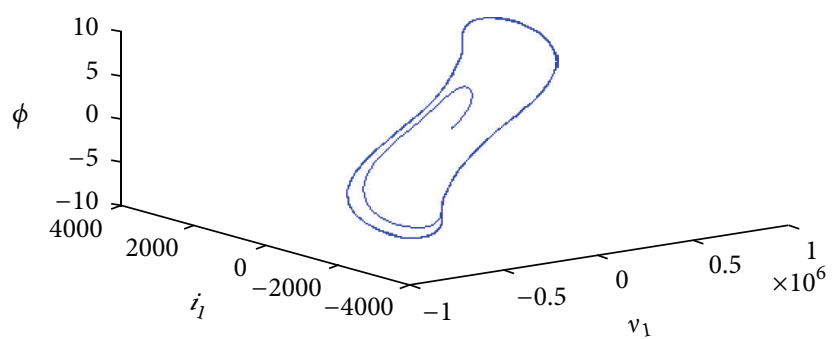

Initial condition ( $\left.\begin{array}{lllll}1 & 1 & 1 & 0 & 0\end{array}\right)$

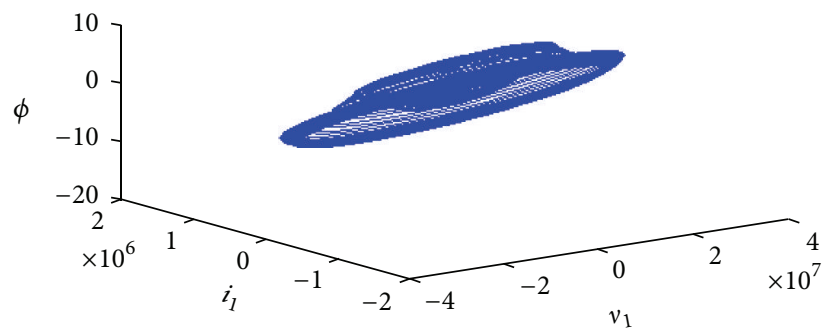

Initial condition $\left(\begin{array}{llll}0.1 & 0.1 & 0.1 & 0.1\end{array}\right)$

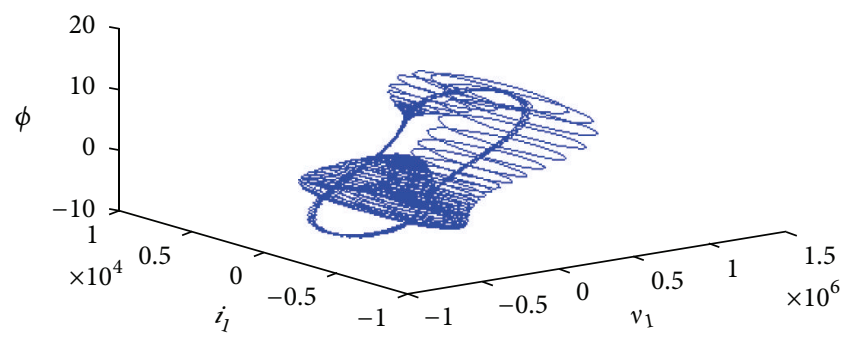

Initial condition $\left(\begin{array}{llll}0 & 0.1 & 0.1 & 0.1\end{array}\right)$

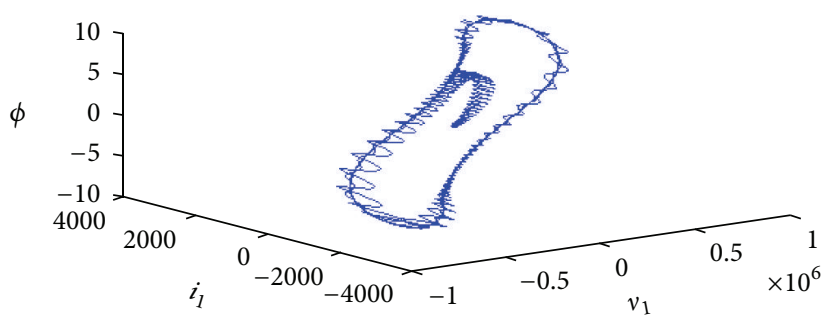

FIGURE 6: 3D Chaotic attractor in different initial conditions.

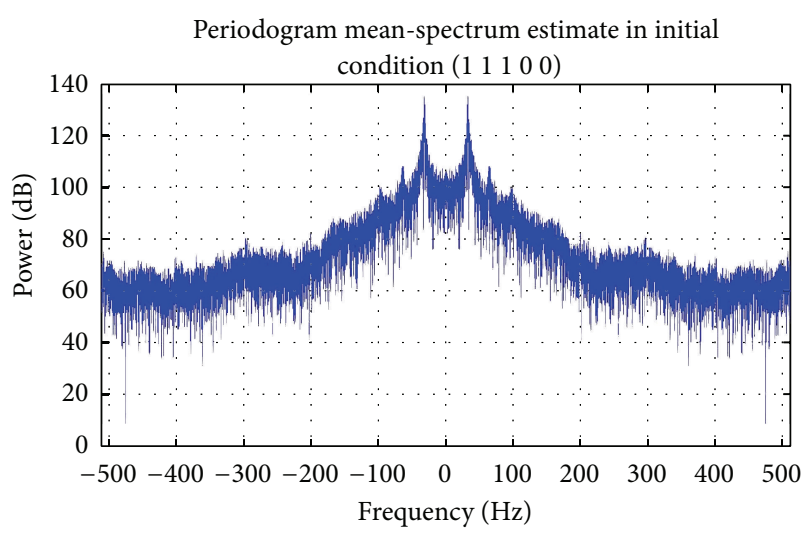

FIGURE 7: The self-power density spectrum chart of the chaotic oscillator.

oxide $\mathrm{TiO}_{2}$ (size $D \approx 10-30 \mathrm{~nm}$ ) sandwiched between two platinum nanowire connections [15].

One of the layers is doped with oxygen vacancies with a high dopant and converted to a semiconductor with low resistance. The second well-known undoped region (doped with a low dopant concentration) has high resistance. The physical model of memristor is shown in Figure $1[15,16]$.

Overall resistance of the elements is equal to two series resistance that both are dependent on the width $(W(t))$ and value of $R_{\mathrm{ON}}$ and $R_{\mathrm{OFF}}$ :

$$
\begin{gathered}
M(x)=R_{\mathrm{ON}}(x(t))+R_{\mathrm{OFF}}(1-x(t)) \\
x(t)=\frac{W(t)}{D}, \quad 0 \leq x(t) \leq 1,
\end{gathered}
$$

where $D$ is the total length, $W(t)$ is the instantaneous width of the semiconductor layer limited to values between zero and
$D(W(t) \in[0, D])$, and $x(t)$ is the length ratio of the doped region to the total length of memristor. $R_{\mathrm{ON}}$ is the resistance in which the total area $(D)$ of the memristor is doping $(W(t)=$ $D)$ and $R_{\mathrm{OFF}}$ is the memristor resistance in which the total area is undoping.

The voltage versus current relation of a charge-controlled memristor is given as follows:

$$
v(t)=\left(R_{\mathrm{ON}}(x(t))+R_{\mathrm{OFF}}(1-x(t))\right) i(t),
$$

where $v(t)$ is the two-terminal voltage of the memristor and $i(t)$ is its current. When an external voltage is cut, the border will remain at its current condition and the value of memristor resistance is preserved which is indicative of memristor memory.

The speed of the movement of the boundary between the doped and undoped regions depends on the resistance of doped area, on the passing current, and on other factors according to the following state equation $[15,16]$ :

$$
\frac{d x}{d t}=k i(t) f(x), \quad k=\frac{\mu_{v} R_{\mathrm{ON}}}{D^{2}},
$$

where $\mu_{v} \approx 10^{-14} \mathrm{~m}^{2} \mathrm{~s}^{-1} \mathrm{~V}^{-1}$ is the so-called dopant mobility and $f(x)$ is the window function that can model the nonlinear dopant drift. The window function can be modeled as a polynomial function $[15,16]$ :

$$
f(x)=1-(2 x-1)^{2 p},
$$

where $p$ is a positive integer [15].

The schematic of the Advance Design System (ADS) software implementation is presented in Figure 2.

A typical characteristic of the memristor is the hysteresis behavior followed by current-voltage domains as plotted in Figure 3. 

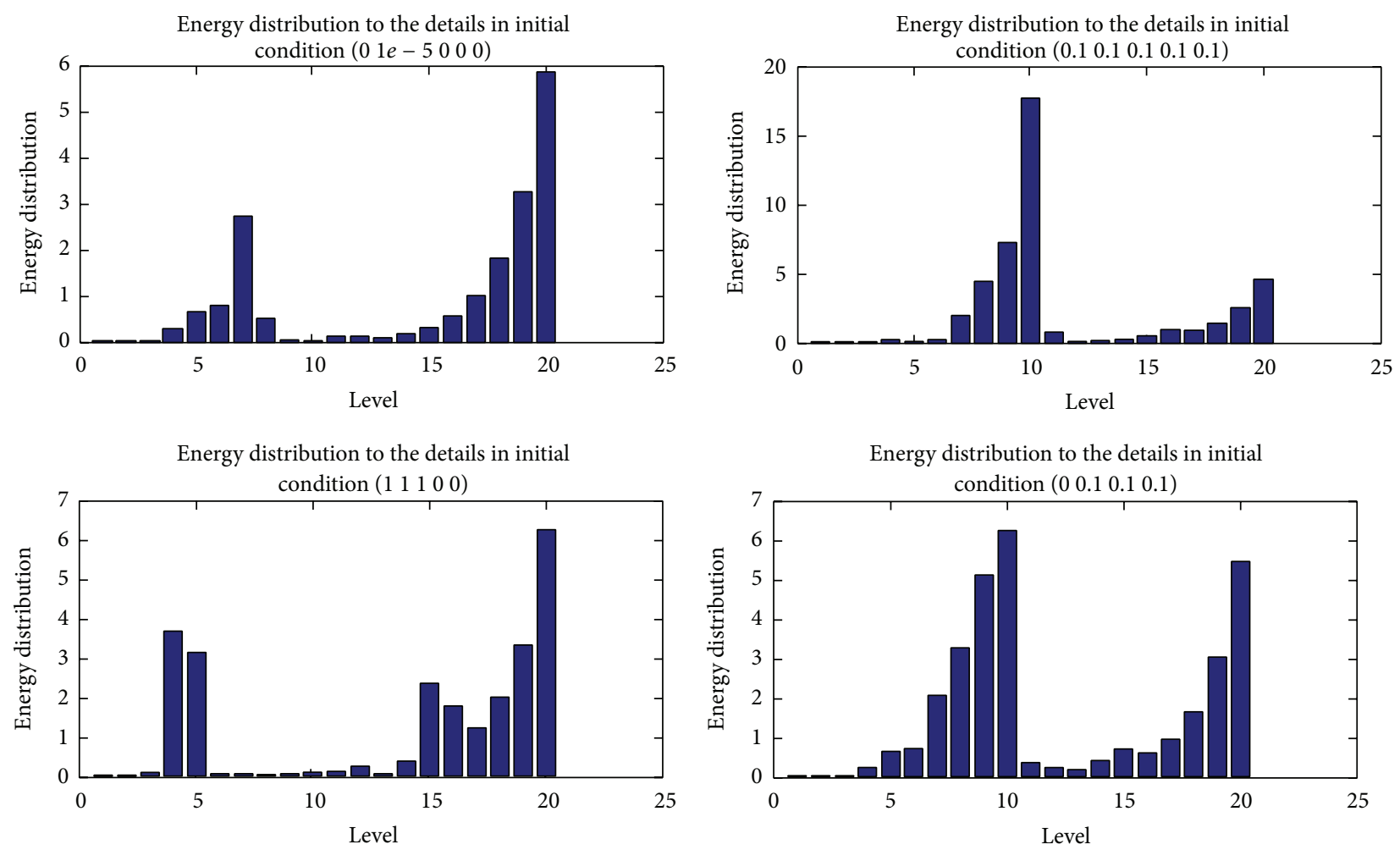

FIGURE 8: Energy distributions in different initial condition of the chaotic oscillator.

\section{Chaotic Oscillator Analysis Based on Memristor}

Figure 4 indicates a chaotic nonlinear oscillator in which a nonlinear resistance is replaced with the memristor. Using the Kirchhoff rules, the dynamic equations describing the circuit are as follows:

$$
\begin{gathered}
\frac{d v_{1}(t)}{d t}=\frac{1}{C_{1}}\left(i_{L_{1}}-W(\varphi(t)) v_{1}(t)\right) \\
\frac{d v_{2}(t)}{d t}=\frac{1}{C_{2}}\left(G_{1} v_{2}(t)-i_{L_{1}}-i_{L_{2}}\right) \\
\frac{d i_{L_{1}}(t)}{d t}=\frac{\left(v_{2}(t)-v_{1}(t)-R i_{L_{1}}\right)}{L_{1}} \\
\frac{d i_{L_{2}}(t)}{d t}=\frac{\left(v_{2}(t)\right)}{L_{2}} \\
\frac{d \varphi}{d t}=v_{1}(t) .
\end{gathered}
$$

The $W(\varphi(t))$ can be given as follows $[12,13]$ :

$$
W(\varphi(t))=\alpha+3 \beta \varphi^{2} .
$$

The circuit parameters are selected as $L_{1}=18 \mathrm{mH}, L_{2}=$ $18 \mathrm{mH}, C_{1}=10 \mathrm{nF}, C_{2}=10 \mathrm{nF}, g=0.25 \times 10^{-2}, R=1 \mathrm{~K}$, $\alpha=0.667 \times 10^{-3}$, and $\beta=0.029 \times 10^{-3}$.
Simulation results indicate that the circuit has a chaotic behavior. Limit cycles for different initial conditions are shown in Figure 5. In order to analyze the chaos more accurately and show sensitivity to the initial conditions, Lyapunov's exponents (LE) of the system are calculated. The existence of a positive Lyapunov's exponent indicates chaos in this circuit [11]. The LEs of the above circuit are calculated as follows: $\lambda_{1}=0.6661, \lambda_{2}=-0.992, \lambda_{3}=-0.661, \lambda_{4}=$ $-0.992, \lambda_{5}=-0.661$. Presence of a positive LE confirms the occurrence of chaotic behavior in the systems. The chaotic attractor can be obtained from the system (9), as depicted in Figure 6.

Lemma 1. The chaotic oscillator based on the memristor proposed in Figure 4 has chaotic behavior because this circuit has one positive Lyapunov's exponent.

\section{Power Density Spectrum Analysis of the Chaotic System}

An effective tool in the study of chaotic behavior is the frequency domain periodic analysis. In periodic signals, the energy is focused on some special frequencies, while in the chaotic behavior the energy in different frequency values is nonzero. Therefore chaotic signals are wideband signals. In deterministic systems, a spectrum having a wideband represents the sign of starting a chaotic behavior [17]. Figure 7 shows the self-power density spectrum of the above circuit. 


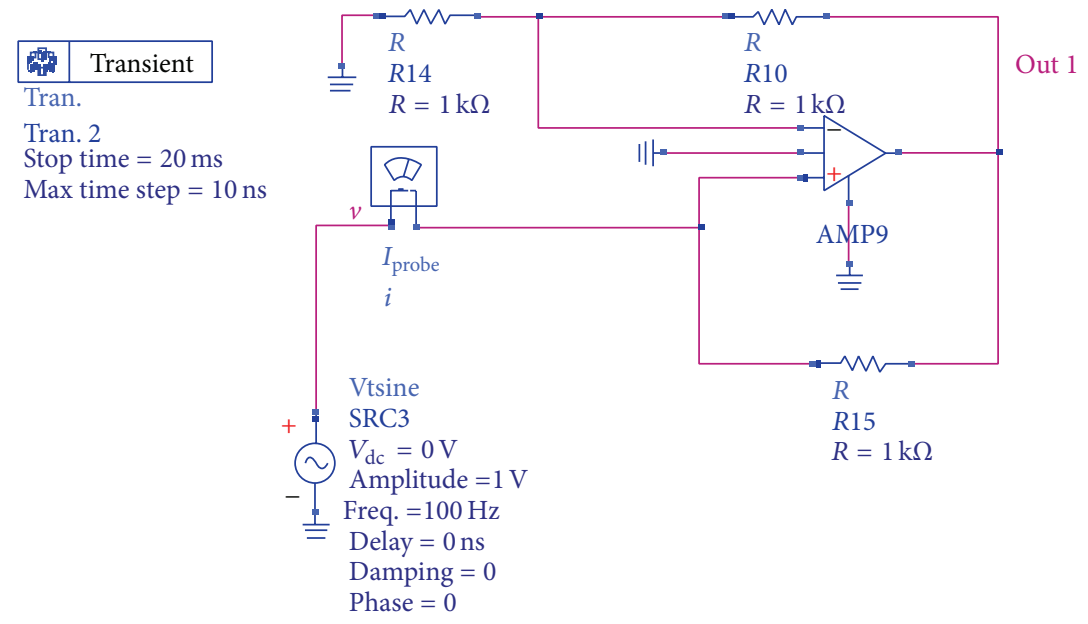

(a)

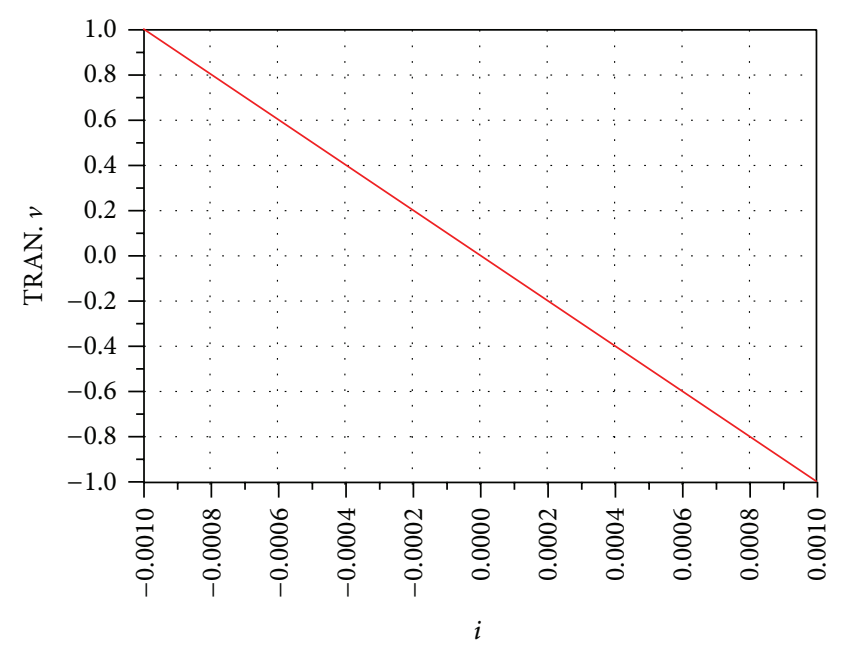

(b)

FIGURE 9: (a) Negative resistance convertor; (b) $v-i$ characteristic of negative resistance convertor.

\section{Energy Distribution Based on the Discrete Wavelet Transform}

In the wavelet analysis, similar to the short-time Fourier transform, a desired signal is multiplied by a wavelet function that plays the role of a window function. In addition, the wavelet transform is also performed separately on different time segments of the signal.

Wavelet is a given function with zero mean and the expansion is conducted in terms of transitions and dilations of the function. Unlike trigonometric polynomials, wavelets in the space are considered locally and thus there is a closer relationship between some functions and their coefficients in the wavelet analysis.

The continuous wavelet transform is defined as follows:

$$
W(a, b)=|a|^{-1 / 2} \int_{-\infty}^{+\infty} x(t) \psi\left(\frac{t-t_{0}}{a}\right) d t,
$$

in which $t_{0}$ and a are transfer and scale parameters.
The transfer concept is exactly similar to a time transfer concept in short-time Fourier transform that clarifies window displacement value and includes transform time information, but, unlike the short-time wavelet transform, there are no frequency parameter in the wavelet transform directly. Instead, there is a scale parameter that is connected conversely with the frequency. And in wavelet instead of frequency transform, the scale parameter is available. In Discrete Wavelet Transform method the signal can be decomposed into different frequency bands. In this method two sets of coefficients are computed: approximation coefficients and detail coefficients. The approximation coefficients are obtained by convolving the signal with the low-pass filter and detail coefficients are obtained by convolving the signal with the high-pass filter for details.

Scaling as a mathematical operator shrinks or expands the signal. Thus, in high scales in which the signal is expanded, we will have details; and in low scales in which the signal is shrunk, we will have generality [18]. 


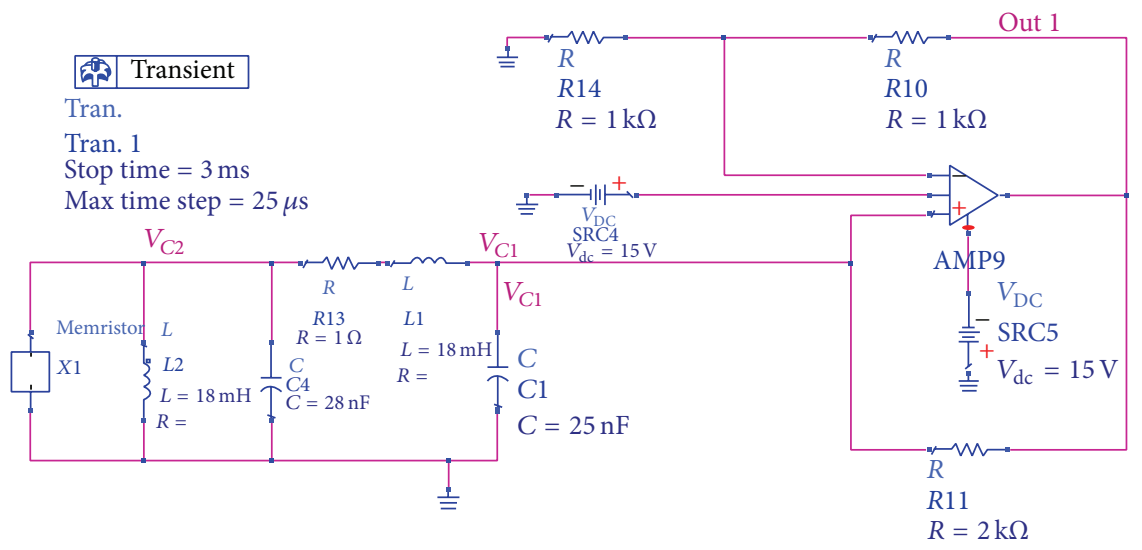

(a)

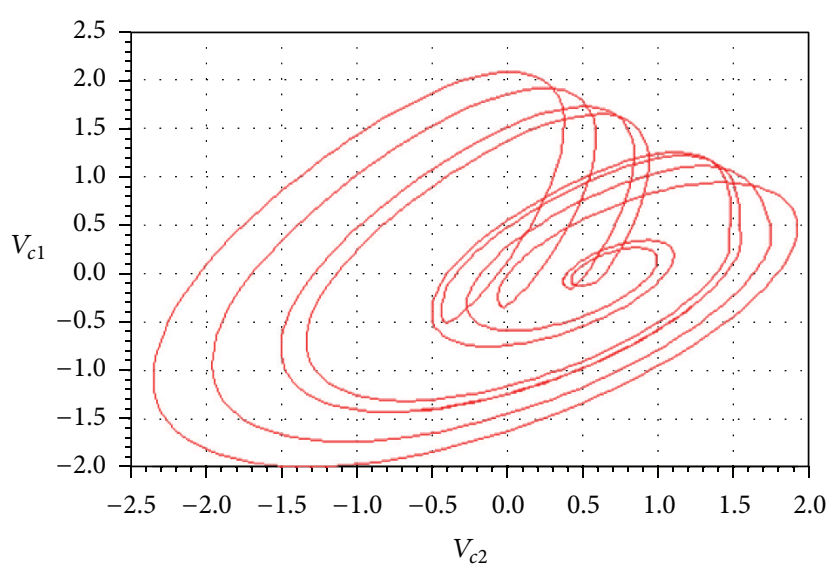

(b)

FIGURE 10: (a) ADS implementation of chaotic oscillator based on memristor; (b) phase plane $V_{c 1}$ versus $V_{c 2}$ from ADS implementation.

In order to analyze more precisely, details and generalities of the signal can be extracted considering the Discrete Wavelet transform. By extracting the coefficient of the signal details using the wavelet transform, energy can be calculated for any detail coefficient as follows:

$$
E\left(d_{i}\right)=\sum_{i=1}^{m}\left|d_{i}\right|^{2},
$$

where $d_{i}$ is the coefficients of details of level $i$, and the total energy can be calculated as follows:

$$
E=\sum_{i=1}^{m} E\left(d_{i}\right) .
$$

The growth rate of energy for each detail is calculated as follows:

$$
\eta_{i}=\frac{E\left(d_{i}\right)}{E} \times 100 .
$$

To illustrate irregular energy distribution of the signal details in each step, entropy is applied. Entropy is the irregularity degree in a system with energy or data. The less is a system regular, the more is the entropy. It is obvious that chaos is a wideband signal. In other words, it can be said that the energy distribution of signal details includes irregular changes in chaos signal [18]. Figure 8 shows distribution of the energy of signal details in each level in chaotic signal under different initial conditions.

\section{ADS Implementation of Chaotic Oscillator Based on Memristor}

Chua's circuit can be realized in variety of ways using standard or custom-made electronic components. Since all of the linear elements (capacitor, resistor, and inductor) are ready available as two-terminal devices, our principal concern here will be with circuitry to realize the negative resistor.

A two-terminal negative resistance convertor can now be produced by connecting three positive resistances around an operation amplifier. An operational amplifier (op-amp) provides us with a real approximation to a voltage-controlled voltage source. Figure 9 shows operation amplifier-based negative resistance convertor and $v-i$ characteristic of opamp negative resistance convertor.

Figure 10 shows an ADS implementation of chaotic oscillator based on memristor and phase plane $V_{c 1}$ versus $V_{c 2}$ from 
ADS implementation. The memristor model implementation is inspired by the charge-controlled memristor implementation discussed in Section 2.

\section{Conclusion}

This paper studies a new structure of the chaotic oscillator based on thememristor. For nonlinear analysis of the memristor chaotic circuit, the Lyapunov exponent method and a new method based on the energy distribution are presented using the Discrete Wavelet Transform (DWT). In this paper, the physical model of memristive element such as the memristor and chaotic circuit based on memristor are implemented using the ADS software.

Since circuit parameters can be continuously tuned in wide ranges, the number of orthogonal chaotic signals can be infinite. This analysis provides a rigorous proof of chaos for memristor circuits.

To summarize, this work attempts to approach nonlinear circuits implemented by the use of memristors. This analysis provides a rigorous proof of chaos for memristor circuits.

\section{Conflict of Interests}

The authors declare that there is no conflict of interests regarding the publication of this paper.

\section{References}

[1] C. Bianca and L. Rondoni, "The nonequilibrium ehrenfest gas: a chaotic model with flat obstacles?” Chaos, vol. 19, no. 1, Article ID 013121, 2009.

[2] C. Bianca, "Weyl-flow and the conformally symplectic structure of thermostatted billiards: the problem of the hyperbolicity," Nonlinear Analysis: Hybrid Systems, vol. 5, no. 1, pp. 32-51, 2011.

[3] I. Djurović and V. Rubežić, "Multiple STFT-based approach for chaos detection in oscillatory circuits," Signal Processing, vol. 87, no. 7, pp. 1772-1780, 2007.

[4] L. Chua, "Memristor-the missing circuit element," IEEE Transactions on Circuit Theory, vol. 18, no. 5, pp. 507-519, 1971.

[5] D. B. Strukov, G. S. Snider, D. R. Stewart, and R. S. Williams, "The missing memristor found," Nature, vol. 453, no. 7191, pp. 80-83, 2008.

[6] J. M. Tour and T. He, "Electronics: the fourth element," Nature, vol. 453, no. 7191, pp. 42-43, 2008.

[7] P. Marks, "Missing memristor makes an appearance," New Scientist, vol. 198, no. 2654, p. 26, 2008.

[8] B. C. Bao, Z. Liu, and J. P. Xu, "Steady periodic memristor oscillator with transient chaotic behaviours," Electronics Letters, vol. 46, no. 3, pp. 228-230, 2010.

[9] B. Muthuswamy and P. Kokate, "Memristor-based chaotic circuits," IETE Technical Review, vol. 26, no. 6, pp. 417-429, 2009.

[10] M. Itoh and L. O. Chua, "Memristor oscillators," International Journal of Bifurcation and Chaos, vol. 18, no. 11, pp. 3183-3206, 2008.

[11] B. Muthuswamy, "Implementing memristor based chaotic circuits," International Journal of Bifurcation and Chaos, vol. 20, no. 5, pp. 1335-1350, 2010.
[12] B. Muthuswamy and L. O. Chua, "Simplest chaotic circuit," International Journal of Bifurcation and Chaos, vol. 20, no. 5, pp. 1567-1580, 2010.

[13] G. Q. Zhong, "Implementation of Chua's circuit with a cubic nonlinearity," IEEE Transactions on Circuits and Systems I, vol. 41, no. 12, pp. 934-941, 1994.

[14] K. Thamilmaran, M. Lakshmanan, and A. Venkatesan, "Hyperchaos in a modified canonical Chua's circuit," International Journal of Bifurcation and Chaos in Applied Sciences and Engineering, vol. 14, no. 1, pp. 221-243, 2004.

[15] H. Wang, X. Wang, C. Li, and L. Chen, "SPICE mutator model for transforming memristor into meminductor," Abstract and Applied Analysis, vol. 2013, Article ID 281675, pp. 1-5, 2013.

[16] Z. Hu, Y. Li, and J. Yu, "Chaotic oscillator based on voltagecontrolled memcapacitor," in Proceedings of the IEEE International Conference on Communications, Circuits and Systems, pp. 824-827, July 2010.

[17] Q. Zhu and S. Liang, "A Method for detecting chaotic vibration based on continuous wavelet transform," International Journa Sensing, Computing and Control, vol. 1, no. 2, pp. 125-132, 2011.

[18] B. Boashash, "Time frequency signal analysis: past, present and future trends," Control and Dynamic Systems, vol. 78, pp. 1-69, 1996. 


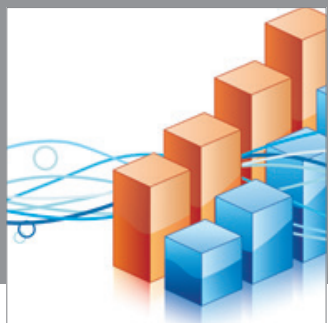

Advances in

Operations Research

mansans

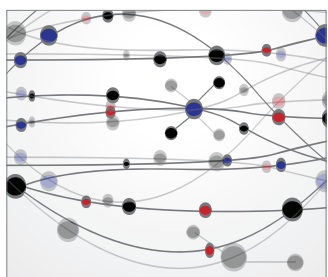

The Scientific World Journal
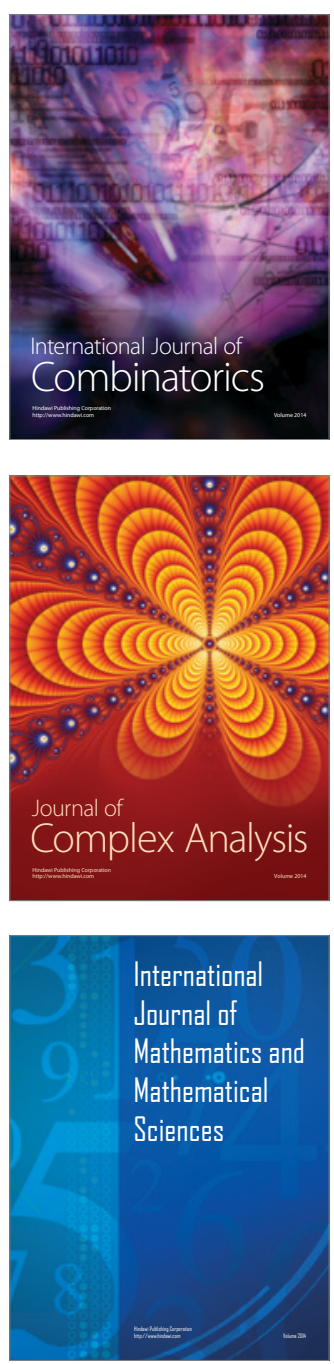
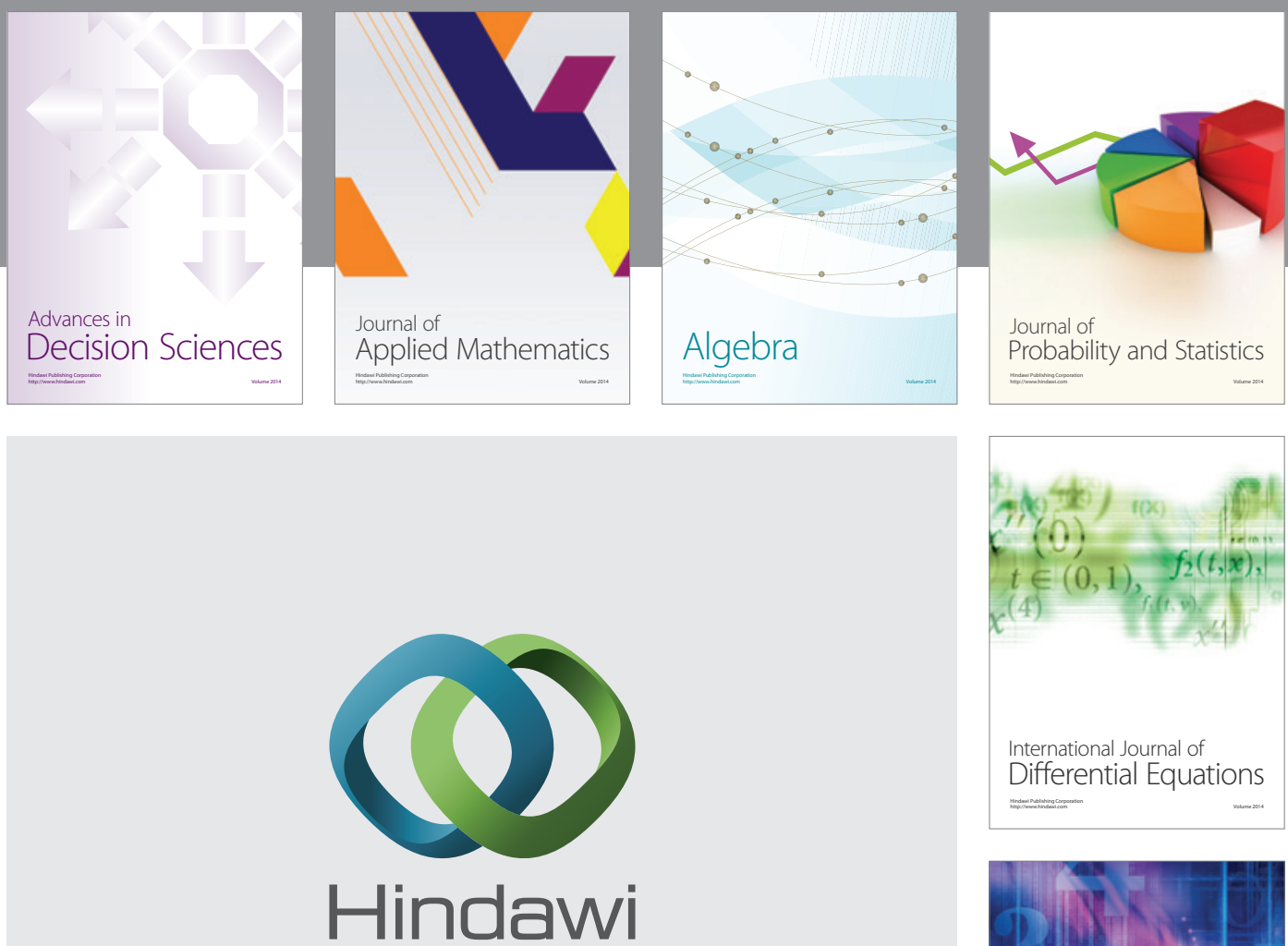

Submit your manuscripts at http://www.hindawi.com
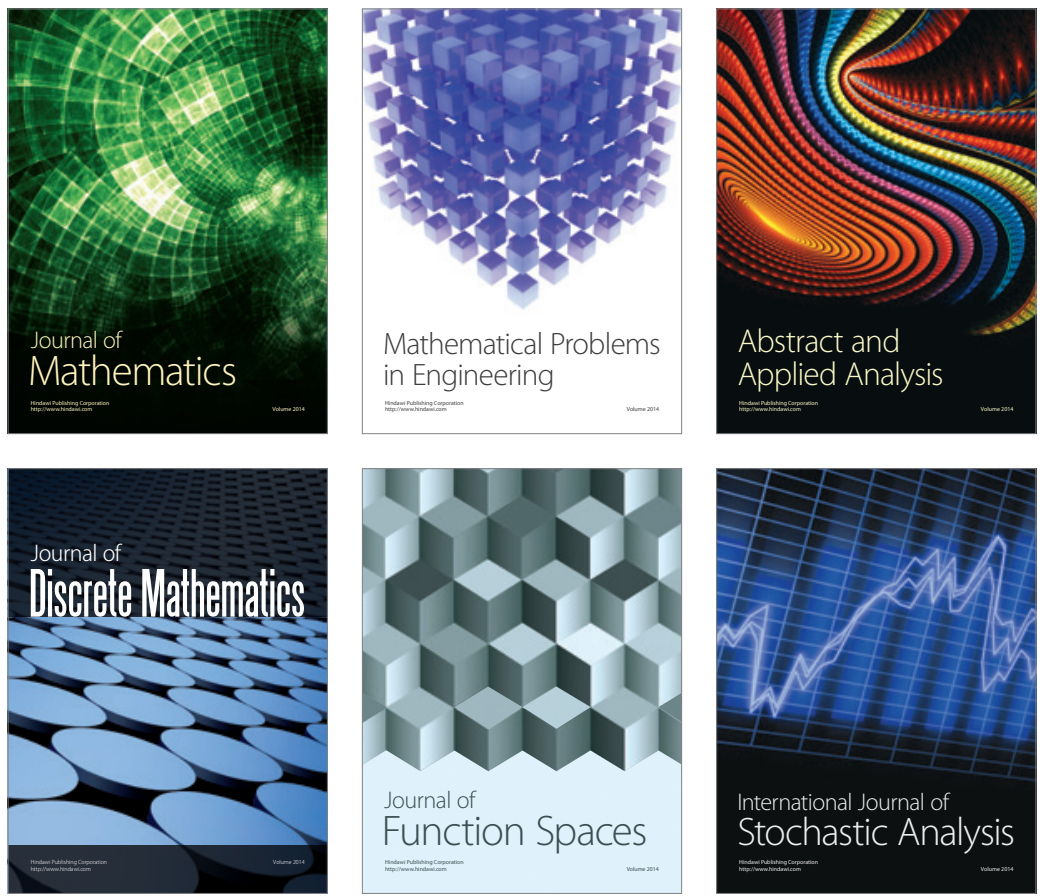

Journal of

Function Spaces

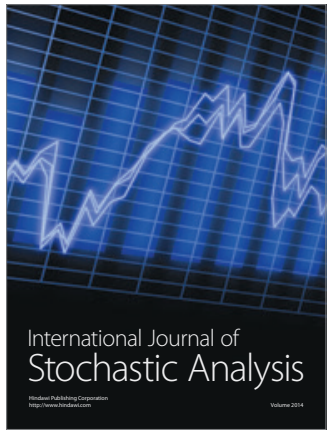

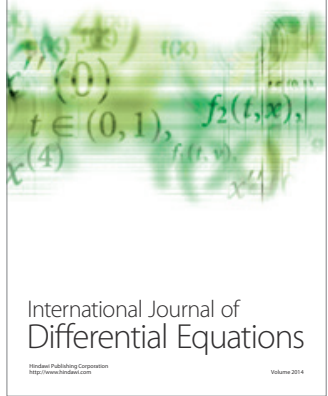
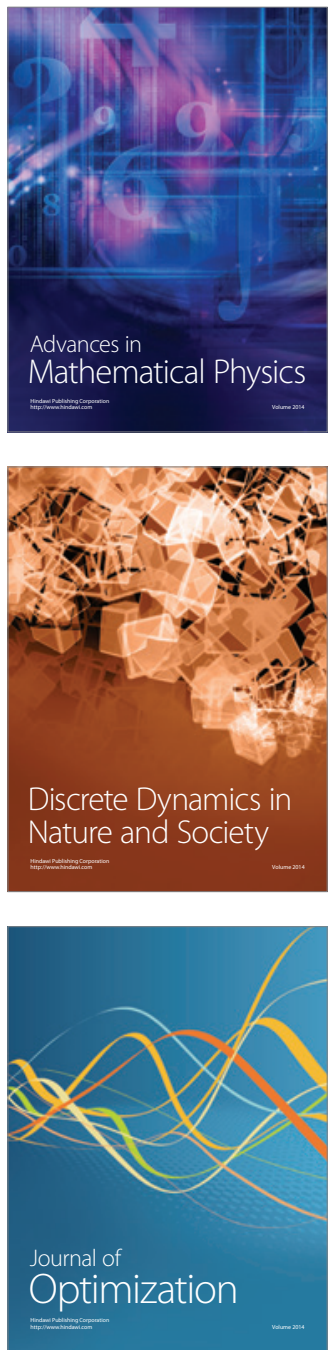